(2) Open Access Full Text Article

ORIGINALRESEARCH

\title{
Moderate-to-Severe Obstructive Sleep Apnea and Cognitive Function Impairment in Patients with COPD
}

This article was published in the following Dove Press journal: International Journal of Chronic Obstructive Pulmonary Disease

\author{
Xiao Lei Zhang ${ }^{1-5}$ \\ Bo $\mathrm{Gao}^{1-3}$ \\ Teng Han ${ }^{1,2}$ \\ Bo Yun Xiang ${ }^{1,2}$ \\ Xin Liu ${ }^{1,2}$ \\ 'Department of Pulmonary and Critical \\ Care Medicine, Center of Respiratory \\ Medicine, China-Japan Friendship \\ Hospital, Beijing, People's Republic of \\ China; ${ }^{2}$ National Clinical Research \\ Center for Respiratory Diseases, Beijing, \\ People's Republic of China; ${ }^{3}$ Capital \\ Medical University, Beijing, People's \\ Republic of China; ${ }^{4}$ The Graduate School \\ of Peking Union Medical College, Chinese \\ Academy of Medical Science and Peking \\ Union Medical College, Beijing, People's \\ Republic of China; ${ }^{5}$ Peking University \\ Health Science Center, Beijing, People's \\ Republic of China
}

Correspondence: Xiao Lei Zhang Department of Pulmonary and Critical Care Medicine, Center of Respiratory Medicine, China-Japan Friendship Hospital, Beijing, People's Republic of China

Fax +86-10-8420-6380

Email yutian728@sina.com
Purpose: Prior studies have indicated that patients with chronic obstructive pulmonary disease (COPD) exhibit significant cognitive defects on neuropsychological testing. Obstructive sleep apnea (OSA) is common in patients with COPD and is associated with reduced cognitive function; however, the combined impact of these two conditions on cognitive function is unknown. The aim of the study was to investigate the impact of OSA on cognitive impairment in patients with COPD.

Methods: Sixty-five stable COPD patients aged over 60 years underwent overnight polysomnography (PSG). Global cognitive functions were evaluated using the Mini-Mental State Examination (MMSE).

Results: Compared to patients with COPD alone, patients with both COPD and OSA performed worse on the MMSE $(25.5 \pm 2.9$ vs $23.5 \pm 3.2 ; \mathrm{p}=0.01)$ and were more likely to

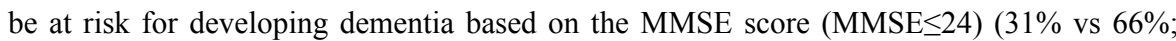
$\mathrm{p}<0.01$ ), independent of key demographic, educational and medical variables known to affect cognitive function in COPD. COPD patients with an apnea hypopnea index (AHI) of $\geq 30$ events/h had lower MMSE scores than those with an AHI of $<15$ events $/ \mathrm{h}$. In addition to age and education level, the severity of nocturnal intermittent hypoxia is an independent predictor of the risk of dementia in patients with COPD $(\mathrm{OR}=1.24,95 \%$ CI 1.04-1.48, $\mathrm{p}=$ $0.02)$.

Conclusion: The current findings indicate that patients with COPD with comorbid OSA may be at greater risk for global cognitive impairment relative to patients with COPD alone. The mechanisms underlying the exaggerated cognitive dysfunction seem to be related to intermittent hypoxia. Further work is needed to understand the impact of OSA on the specific domains of cognitive impairment and the therapeutic implications of OSA in COPD.

Keywords: chronic obstructive pulmonary disease, obstructive sleep apnea, cognitive function

\section{Introduction}

Chronic obstructive pulmonary disease (COPD) is considered a systemic disease with multiple extrapulmonary comorbidities, which may exert a significant impact on the natural courses of disease. ${ }^{1}$ There is increasing evidence that cognitive impairment exists in patients with COPD. ${ }^{2-5}$ A preliminary study by Villeneuve et al found that mild cognitive impairment (MCI) was identified in approximately one-third of patients with moderate-to-severe COPD, predominantly manifesting as attention and executive dysfunction. ${ }^{2}$ A populated-based, prospective-cohort study 
by Singh et al found that COPD was associated with an increased risk of $\mathrm{MCI}$, and there was a dose-response relationship between COPD duration and the risk of MCI. ${ }^{5}$ The mechanisms for cognitive decline in COPD are multifactorial, and hypoxemia, hypercapnia, systemic inflammation and vascular comorbidities may be the main causes; however, it is unlikely that these mechanisms are sufficient to explain cognitive impairment in COPD. ${ }^{6,7}$

Obstructive sleep apnea (OSA) is characterized by recurrent, partial or total collapse of the upper airway with resultant sleep structure abnormalities and nocturnal hypoxemia. Recent reports have demonstrated that patients with OSA scored poorly on measures of memory, attention and executive function, and it may increase the risk of dementia in elderly individuals. ${ }^{8-10}$ Studies with transcranial Doppler found that OSA is associated with rapid, potentially harmful changes in cerebral blood flow, and inadequate cerebral perfusion was more prominent during sequences of repetitive apneas. ${ }^{11}$ Neuroimaging studies indicated that neural damage, including axons and white matter, as well as substantial gray matter loss, were observed in several cortical regions among OSA patients, which was consistent with the deficits in different aspects of cognitive function. ${ }^{12}$ Nocturnal hypoxemia and sleep fragmentation are considered the underlying mechanisms for cognitive impairment in OSA. ${ }^{13}$

OSA is common in patients with COPD and adversely impacts quality of life. ${ }^{14}$ Patients with OSA and COPD (overlap syndrome) may develop more severe nocturnal hypoxia than those with OSA or COPD alone. Olaithe et al studied the separate effects of untreated OSA, COPD and sleep deprivation on cognitive function with a systematic review and meta-analysis and they found that COPD and OSA shared some common cognitive deficits (executive function, memory, attention, language abilities and psychomotor function), which suggests a core role for hypoxia. ${ }^{15}$ Thakur et al reported an association between hypoxia and cognitive impairment as determined by the MMSE and a reduced risk for cognitive impairment in patients receiving supplemental oxygen. ${ }^{16}$ Although not previously examined, it is possible that hypoxia-related mechanisms may simultaneously and/or synergistically exert an influence on cognitive dysfunction in patients with combined OSA and COPD, and sleep apnea may be another important contributor to cognitive defects in COPD. For patients with COPD, adequate cognitive functioning is essential to understand the nature of the disease, to adhere to treatment, and to lead a better quality of life.
Cognitive dysfunction may also be related to increased morbidity and mortality. Given the high prevalence of OSA among COPD patients and its independent impact on cognitive function, the objective of this research was to study the effect of OSA on cognitive impairment in patients with COPD.

\section{Materials and Methods Patient Population}

This cross-sectional study was performed at China-Japan Friendship Hospital from September 2015 to August 2019. Consecutive COPD patients over 60 years old were recruited for this study. The diagnosis of COPD was made according to the Global Initiative for Chronic Obstructive Lung Disease (GOLD) guidelines. ${ }^{14}$ The exclusion criteria included acute exacerbation in the past two months and a history of neurological problems (e.g., Alzheimer's disease, severe cerebrovascular disease and severe head injury), history of significant psychological disorders (e.g., substance abuse, bipolar disorder and schizophrenia), mental retardation, and history of noninvasive positive airway pressure (NIPAP) or nocturnal oxygen therapy at home. This study was conducted in accordance with the Declaration of Helsinki. The China-Japan Friendship Hospital Ethics Committee approved the protocol, and all patients provided informed consent.

\section{Pulmonary Function Tests, Arterial Blood Gas (ABG) Analysis and Clinical Questionnaires}

Pulmonary function tests were measured according to the criteria of the American Thoracic Society/European Respiratory Society. ${ }^{18}$ Daytime ABG analysis was performed on room air after $15 \mathrm{~min}$ of rest following pulmonary function tests. The Epworth Sleepiness Scale (ESS) was used to evaluate the tendency to doze off or fall asleep during the day, with a score $>10$ indicating excessive daytime sleepiness. ${ }^{19}$ The Hospital Anxiety and Depression Scale was used to evaluate anxiety and depression simultaneously. ${ }^{20}$ The COPD Assessment Test (CAT) was used to assess health-related quality of life (HRQL), with higher scores indicating worse HRQL. ${ }^{21}$

\section{Cognitive Function Evaluation}

The Mini-Mental State Examination (MMSE) was used to measure global cognitive function. It is composed of questions about different cognitive abilities, such as attention, 
concentration, orientation, sequence, visual-spatial abilities, understanding, reading aloud and verbal learning, memory and repetition. The MMSE is a screening test for cognitive impairment, with a maximum score of 30 . A score $\geq 28$ is considered to be normal, a score of $25-27$ is considered to be mild impairment, and a score $\leq 24$ indicates a high risk for dementia. ${ }^{22}$ A specific trained research staff member administered the MMSE scale the morning after the sleep study.

\section{Polysomnographic Study}

Full overnight polysomnography (PSG) (Alice 6, Philips, USA) was conducted with the patients breathing room air in the sleep center. All data were analyzed by experienced PSG technologists according to the updated 2012 American Academy of Sleep Medicine (AASM) criteria. ${ }^{23}$ OSA was defined as an apnea hypopnea index (AHI) of $\geq 15$ events $/ \mathrm{h} .{ }^{24}$ A desaturation of $\geq 3 \%$ for $\geq 10$ seconds and $\leq 3$ minutes was defined as a desaturation event. The oxygen desaturation index (ODI) was defined as the number of desaturation events per hour.

\section{Statistical Analysis}

Data are presented as the mean \pm standard deviation for numerical variables with a normal distribution or as the median and interquartile range for data with a skewed distribution. Categorical variables are expressed as frequencies and proportions. Comparisons of continuous variables between groups were determined by the unpaired $t$-test, one-way ANOVA or Mann-Whitney $U$-test, and the chi-square test or Fisher's exact test was used for categorical data. Univariable logistic regression analysis was used to evaluate the association between possible predictors and risk of dementia. Predictor variables with $\mathrm{p}<0.10$ according to the univariable analysis were selected for multivariable regression analysis. A value of $p<0.05$ was considered statistically significant. All analyses were conducted with SPSS 22.0 (SPSS Inc., Chicago, Illinois, United States).

\section{Results}

\section{Study Population Characteristics}

The study population included 65 patients with mainly moderate-to-severe COPD. The baseline characteristics are summarized in Table 1. The demographics, sex distribution, education level, smoking status, mean ESS score, pulmonary function parameters, resting blood-gas levels, and anxiety and depression scores were comparable between patients with and without OSA. However, COPD patients with sleep apnea were more likely to have hypertension and performed worse on the CAT score.

\section{PSG in Patients with and without OSA}

The PSG parameters are shown in Table 2. The AHI, hypopnea index, ODI, and arousal index were higher, while the lowest $\mathrm{SpO}_{2}$ was lower in patients with OSA than in patients without OSA. The OSA group had more sleep time with $\mathrm{SpO}_{2}$ below 90\% (T90). The total sleep time, median sleep efficiency, sleep structure and mean $\mathrm{SpO}_{2}$ were comparable between patients with and without OSA.

\section{Cognitive Function}

The average MMSE score of the whole study population was 24.7 \pm 3.2 . Among the COPD patients, 30 (46\%) had an MMSE score below 24, 23 (35\%) had an MMSE score between 25 and 27, and 12 (18\%) had an MMSE score above 28. COPD patients with OSA performed significantly worse on the MMSE than COPD patients without OSA. Using an MMSE score of 24 points as the cutoff value for risk for dementia, $66 \%$ of patients with OSA had a risk of dementia compared to $31 \%$ of patients in the COPD alone group. Using an MMSE score of 25-27 points as the indicator for MCI, the prevalence of MCI was comparable between the two groups (Table 1).

\section{Factors Associated with Risk of Dementia}

For COPD patients without OSA, the risk of dementia was associated with older age and lower educational level. In the multivariable regression analysis, with the risk of dementia as the dependent variable and age, educational level, and ODI as independent variables, age and educational level were independently associated with the risk of dementia. For COPD patients with OSA, the risk of dementia was associated with older age, lower educational level, and higher AHI and ODI values according to univariable logistic regression analysis. In the multivariable regression analysis, with the risk of dementia as the dependent variable and age, educational level, AHI and ODI as independent variables, age, educational level and ODI were significantly associated with a risk of dementia.

For the whole patient population, the risk of dementia was associated with older age, lower educational level, and higher AHI and ODI values according to univariable regression analysis. Patients with severe OSA (AHI $\geq 30$ 
Table I Baseline Patient Characteristics

\begin{tabular}{|c|c|c|c|}
\hline Variables & COPD+OSA $(n=29)$ & COPD $(n=36)$ & p-value \\
\hline Age, years & $70.4 \pm 7.8$ & $73.5 \pm 9.6$ & 0.16 \\
\hline Male/Female & $24 / 5$ & $27 / 9$ & 0.45 \\
\hline $\mathrm{BMI}, \mathrm{kg} / \mathrm{m}^{2}$ & $22.3 \pm 4.7$ & $23.1 \pm 4.7$ & 0.86 \\
\hline Education, years & $8.3 \pm 3.7$ & $8.0 \pm 4.4$ & 0.38 \\
\hline Smoking status & & & 0.67 \\
\hline Current Smokers (n, \%) & $10(34 \%)$ & $9(25 \%)$ & \\
\hline Former smokers $(\mathrm{n}, \%)$ & 14 (48\%) & $21(58 \%)$ & \\
\hline Never-smokers (n, \%) & $5(17 \%)$ & $6(17 \%)$ & \\
\hline Pack-years & $48(32-76)$ & $50(35-80)$ & 0.65 \\
\hline Hypertension (n, \%) & $22(76 \%)$ & $16(44 \%)$ & 0.01 \\
\hline Diabetes (n, \%) & $10(27 \%)$ & $8(28 \%)$ & 0.98 \\
\hline IHD (n, \%) & II(3I\%) & $6(21 \%)$ & 0.37 \\
\hline Cerebral infarction (n, \%) & $7(19 \%)$ & $4(14 \%)$ & 0.55 \\
\hline GOLD severity, I, II/III, IV & $14 / 15$ & $18 / 18$ & 0.16 \\
\hline $\mathrm{FEV}_{1}, \%$ predicted & $54.3 \pm 18.9$ & $51.8 \pm 18.9$ & 0.24 \\
\hline FVC, \% predicted & $79.1 \pm 22.9$ & $80.6 \pm 18.0$ & 0.78 \\
\hline $\mathrm{FEV}_{\mathrm{l}} / \mathrm{FVC}$, ratio & $53.1 \pm 10.0$ & $49.6 \pm 12.7$ & 0.26 \\
\hline TLC, \% predicted & $84.8 \pm 17.9$ & $82.7 \pm 18.6$ & 0.67 \\
\hline $\mathrm{RV} / \mathrm{TLC}, \%$ & $55.0 \pm 12.2$ & $53.3 \pm 10.3$ & 0.58 \\
\hline DLCO, \% predicted & $63.3 \pm 24.7$ & $59.7 \pm 17.8$ & 0.53 \\
\hline $\mathrm{PaO}_{2}(\mathrm{mmHg})$ & $77.7 \pm 16.8$ & $77.1 \pm 20.2$ & 0.91 \\
\hline $\mathrm{PaCO}_{2}(\mathrm{mmHg})$ & $43.2 \pm 5.1$ & $42.6 \pm 7.0$ & 0.75 \\
\hline Anxiety score & $5(3-8)$ & $5(2-7)$ & 0.59 \\
\hline Depression score & $4.5 \pm 4.3$ & $3.6 \pm 4.0$ & 0.41 \\
\hline ESS & $5.2 \pm 4.0$ & $4.4 \pm 3.9$ & 0.42 \\
\hline CAT & $21.7 \pm 4.7$ & $18.2 \pm 5.5$ & $<0.01$ \\
\hline Beta-2 agonist use, $\mathrm{n}(\%)$ & $18(62 \%)$ & $22(61 \%)$ & 0.94 \\
\hline Anticholinergic use, $\mathrm{n}(\%)$ & $21(72 \%)$ & $27(75 \%)$ & 0.81 \\
\hline Inhaled corticosteroid use, $\mathrm{n}(\%)$ & I4(48\%) & $19(53 \%)$ & 0.72 \\
\hline Mini-Mental State Examination & $23.5 \pm 3.2$ & $25.5 \pm 2.9$ & $0.01 *$ \\
\hline Risk for dementia. n (\%) & $19(66 \%)$ & II(3I\%) & $<0.01$ \\
\hline Mild cognitive dysfunction, n (\%) & $7(24 \%)$ & $16(44 \%)$ & 0.09 \\
\hline No cognitive dysfunction, n (\%) & $3(10 \%)$ & $9(25 \%)$ & 0.13 \\
\hline
\end{tabular}

Notes: Data are expressed as mean \pm standard deviation, median and interquartile range, or count (percentage). $* P<0.05$ for all levels of cognitive function between the 2 groups according to Chi Squared test.

Abbreviations: OSA, obstructive sleep apnea; COPD, chronic obstructive pulmonary disease; BMI, body mass index; IHD, ischemic heart disease; FEV forced expiratory volume in I s; FVC, forced vital capacity; GOLD, Global Initiative for COPD; RV, residual volume; TLC, total lung capacity; DLCO, diffusion capacity of the lung for carbon monoxide; $\mathrm{PaO}_{2}$, arteria oxygen partial pressure; $\mathrm{PaCO}_{2}$, arteria carbon dioxide partial pressure; ESS, Epworth Sleepiness Scale; CAT, COPD assessment test.

events/h) had lower MMSE scores than those with an $\mathrm{AHI}<15$ events/h $(22.6 \pm 1.6 \quad$ vs. $25.5 \pm 2.9, \quad \mathrm{p}=0.008$, Figure 1A). The severity of airflow obstruction (Figure 1B), comorbidities, anxiety and depression, CAT score, excessive daytime sleepiness, sleep time, sleep architecture, arousal index, lowest $\mathrm{SpO}_{2}$ and TST90 were not significantly associated with a risk of dementia. In the multivariable regression analysis, with the risk of dementia as the dependent variable and age, educational level, the presence of hypertension, AHI, and ODI as independent variables, age, educational level and ODI were significantly associated with a risk of dementia (Table 3 ).

\section{Discussion}

COPD is a multicomponent disease with a variety of extrapulmonary comorbidities, which may impact the prognosis and increase the risk of mortality. Multidimensional assessment and personalized interventional strategies are key to optimal disease management. It has been documented that patients with COPD may manifest different degrees of 
Table 2 Polysomnography Parameters

\begin{tabular}{|l|l|l|l|}
\hline Variables & $\begin{array}{l}\text { COPD+OSA } \\
(\mathbf{n}=\mathbf{2 9})\end{array}$ & $\begin{array}{l}\text { COPD } \\
\text { (n=36) }\end{array}$ & p-value \\
\hline TST, min & $318 \pm 72$ & $339 \pm 83$ & 0.29 \\
Sleep efficiency, \% & $72.1 \pm 13.5$ & $68.9 \pm 11.9$ & 0.31 \\
Arousal index, events/h & $30.1 \pm 14.6$ & $20.0 \pm 10.4$ & $<0.01$ \\
\hline Sleep architecture, \% TST & & & \\
$\quad$ Stage NI & $16.3 \pm 9.8$ & $14.4 \pm 9.2$ & 0.42 \\
Stage N2 & $57.2 \pm 13.2$ & $54.9 \pm 14.1$ & 0.50 \\
Stage N3 & $10.5 \pm 8.3$ & $13.9 \pm 11.6$ & 0.19 \\
Stage R & $15.9 \pm 11.3$ & $16.8 \pm 11.9$ & 0.76 \\
\hline AHI, events/h & $29.3 \pm 11.4$ & $7.6 \pm 4.1$ & $<0.01$ \\
Al, events/h & $5.2 \pm 7.1$ & $4.6 \pm 6.9$ & 0.73 \\
HI, events/h & $24.1 \pm 12.0$ & $3.0 \pm 4.4$ & $<0.01$ \\
Mean SpO,$\%$ & $92.9 \pm 3.1$ & $93.6 \pm 2.6$ & 0.42 \\
Lowest SpO,$\%$ & $78.9 \pm 7.9$ & $85.0 \pm 6.1$ & $<0.01$ \\
TST90 (\%) & $17.3 \pm 15.7$ & $7.8 \pm 14.6$ & 0.01 \\
ODI ( $\geq 3 \%)$, events/h & $20.8 \pm 11.5$ & $5.6 \pm 5.0$ & $<0.01$ \\
\hline
\end{tabular}

Note: Data are expressed as mean \pm standard deviation.

Abbreviations: OSA, obstructive sleep apnea; $\mathrm{AHI}$, apnoea hypopnoea index; $\mathrm{Al}$, apnea index; $\mathrm{HI}$, hypopnea index; $\mathrm{SpO}_{2}$, pulse oxygen saturation; TST, total sleep time; $\mathrm{R}$, rapid eye movement; ODI, oxygen desaturation index with oximetry recording; TST90, percentage of total sleep time spent with oxyhemoglobin saturation below $90 \%$.

cognitive impairment, which may exert damaging impacts on many facets of patient health status and therapeutic outcomes. Sleep apnea is also known to have adverse impacts on cognitive function, including attention, memory retrieval, and executive functioning. OSA is common in patients with COPD; however, there is a lack of scientific literature investigating the possible additive effects of OSA on cognitive function in patients with COPD. Our study is the first, to our knowledge, to report on sleep apnea and the risk of cognitive impairment in patients with COPD. In the current study, the prevalence of cognitive impairment was high among patients with COPD. Patients with both COPD and OSA showed poorer cognitive functioning than patients with COPD alone, independent of key demographic, educational and medical variables known to affect cognitive function in COPD. Additionally, in addition to age and education level, the severity of nocturnal intermittent hypoxia is an independent predictor of the risk of dementia in patients with COPD.

The brain is vulnerable to the systemic effects of COPD and increasing evidence has shown cognitive deterioration in patients with COPD. It is documented that the prevalence of cognitive impairment varies from $12 \%$ to $88 \%$ in patients with COPD, according to the study population and neuropsychological assessment. ${ }^{25}$ In our patient population, which included older COPD patients who were recruited from a tertiary referral hospital with mainly moderate-to-severe disease severity, $35 \%$ of patients had MCI, and $46 \%$ had a risk of dementia, which implied a high frequency of cognitive decline among patients with advanced COPD. This high prevalence of cognitive impairment in our COPD patients may be due to the relatively older age and lower educational level of our patients. In the present study, the risk of dementia was higher in patients with OSA than in patients without OSA, while the prevalence of MCI was comparable between the two groups. The MMSE, which is one of the most widely used screening tests for the evaluation of global cognitive function, was used in this investigation to measure cognitive functioning in COPD. A meta-analysis of thirty-four dementia studies and five MCI studies reported that the MMSE had good accuracy in identifying dementia from normal aging. In memory clinic settings, the sensitivity (Se) and specificity (Sp) of the MMSE for dementia are $79.8 \%$ and $81.3 \%$, respectively, and in community settings, the $\mathrm{Se}$ and $\mathrm{Sp}$ are $85.1 \%$ and $85.6 \%$, respectively. However, the MMSE had limited ability to discriminate
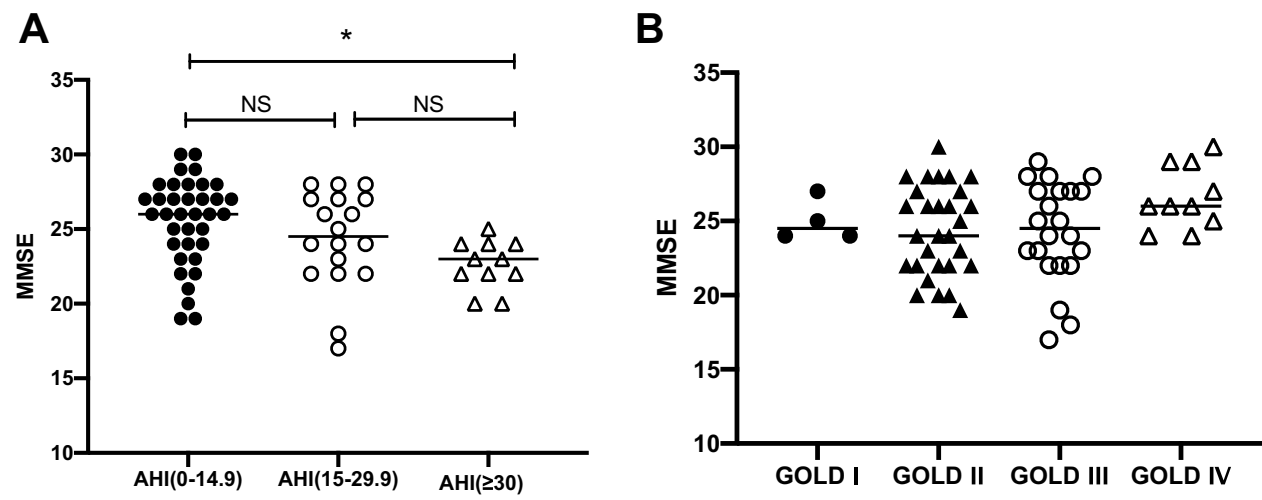

Figure I (A) The comparison of MMSE scores among patients with different severities of OSA. *Versus patients with an AHI<15 events/h, $\mathrm{P}<0.05$. (B) The comparison of MMSE scores among patients with different severities of airflow obstruction.

Abbreviations: AHI, apnoea hypopnoea index; MMSE, Mini-Mental State Examination; GOLD, Global Initiative for COPD. 
Table 3 Possible Predictors of Risk of Dementia in Patients with COPD

\begin{tabular}{|c|c|c|c|}
\hline & Total $(n=65)$ & COPD+OSA $(n=29)$ & COPD $(n=36)$ \\
\hline & OR $(95 \% \mathrm{Cl}) \mathrm{p}$ & OR $(95 \% \mathrm{Cl}) \mathrm{p}$ & OR $(95 \% \mathrm{Cl}) \mathrm{p}$ \\
\hline Age, years & $1.08 \quad 1.02-1.150 .02$ & I.I5 I.0I-I.30 0.03 & $1.241 .06-1.45<0.01$ \\
\hline Male sex & $1.760 .53-5.810 .36$ & $1.330 .18-9.660 .78$ & $0.730 .32-1.670 .45$ \\
\hline Education & $0.450 .26-0.79<0.01$ & $0.240 .08-0.700 .01$ & $0.340 .14-0.840 .02$ \\
\hline Pack-years & $0.770 .23-2.630 .68$ & $1.690 .89-3.190 .11$ & $0.630 .22-2.050 .48$ \\
\hline Hypertension & $0.390 .13-1.150 .09$ & $0.960 .91-1.210 .29$ & $0.580 .12-1.370 .17$ \\
\hline Diabetes & $0.820 .30-2.240 .70$ & $1.050 .96-1.140 .28$ & $0.720 .12-4.160 .71$ \\
\hline IHD & $\begin{array}{llll}0.80 & 0.25-2.5 & 0.70\end{array}$ & $0.970 .93-1.010 .37$ & $1.270 .28-2.3 \mid 0.52$ \\
\hline Cerebral infarction & $0.390 .11-1.370 .14$ & $0.480 .06-3.630 .48$ & $1.020 .98-1.060 .29$ \\
\hline $\mathrm{FEV}_{\mathrm{l}}, \%$ predicted & I.01 $0.98-1.040 .42$ & $0.980 .94-1.020 .30$ & $1.040 .99-1.090 .16$ \\
\hline Anxiety & $0.980 .84-1.140 .76$ & $0.980 .78-1.240 .87$ & $0.940 .74-1.200 .60$ \\
\hline Depression & $0.990 .87-1.110 .83$ & $0.990 .83-1.200 .99$ & $0.920 .74-1.150 .47$ \\
\hline CAT & $0.990 .91-1.090 .91$ & I.II $0.92-1.330 .28$ & $0.970 .85-1.100 .62$ \\
\hline ESS & $1.040 .62-1.760 .87$ & $1.070 .96-1.190 .22$ & $1.120 .83-1.500 .46$ \\
\hline $\mathrm{AHI}$ & I.I7 I.09-1.27<0.0I & I.I8 $1.03-1.340 .02$ & $1.190 .95-1.500 .16$ \\
\hline ODI & I.26 I.I3-1.40<0.0I & 1.37 I. $10-1.70<0.01$ & $1.230 .97-1.500 .09$ \\
\hline Mean $\mathrm{SPO}_{2}, \%$ & $1.070 .89-1.280 .47$ & $0.940 .68-1.290 .70$ & I.II $0.87-1.420 .40$ \\
\hline Lowest $\mathrm{SPO}_{2}, \%$ & $0.990 .93-1.070 .88$ & $0.960 .85-1.070 .45$ & $1.080 .94-1.230 .30$ \\
\hline TST90 & $0.980 .96-1.010 .10$ & I.01 $0.95-1.070 .79$ & $0.980 .94-1.010 .19$ \\
\hline TST, min & $0.990 .97-1.030 .73$ & $0.940 .78-1.120 .47$ & $0.960 .82-1.130 .62$ \\
\hline Arousal index & $1.030 .94-1.120 .59$ & I.04 0.97-I.II 0.25 & $1.060 .99-1.130 .11$ \\
\hline Sleep efficiency, \% & $0.970 .8 \mathrm{I}-\mathrm{I} .120 .7 \mathrm{I}$ & $1.060 .97-1.150 .20$ & $0.980 .93-1.020 .32$ \\
\hline Stage NI, \% TST, & $1.020 .8 \mathrm{I}-\mathrm{I} .350 .59$ & $1.680 .89-3.190 .11$ & I.03 0.98-I.08 0.30 \\
\hline Stage N2, \% TST & $0.960 .90-1.160 .79$ & $1.030 .86-1.190 .91$ & $0.980 .85-1.130 .80$ \\
\hline Stage N3, \% TST & $0.980 .92-1.070 .17$ & $0.980 .95-1.010 .29$ & $0.960 .89-1.040 .36$ \\
\hline Stage R, \% TST & $1.030 .81-1.190 .51$ & $0.940 .78-1.120 .47$ & $1.060 .95-1.180 .38$ \\
\hline \multicolumn{4}{|l|}{ Adjusted analysis } \\
\hline & OR $(95 \% \mathrm{Cl}) \mathrm{p}$ & OR $(95 \% \mathrm{Cl}) \mathrm{p}$ & OR $(95 \% \mathrm{Cl}) \mathrm{p}$ \\
\hline Age, years & I.10 I.0I-I.2I 0.04 & $1.26 \quad 1.05-1.500 .01$ & $1.231 .04-1.460 .01$ \\
\hline Education & $0.380 .14-0.980 .04$ & $0.260 .08-0.890 .03$ & $0.370 .13-0.860 .04$ \\
\hline $\mathrm{AHI}$ & $1.060 .99-1.190 .08$ & $1.070 .94-1.260 .27$ & - \\
\hline ODI & I.24 I.04-1.48 0.02 & I.38 $1.02-1.870 .04$ & $1.030 .98-1.080 .22$ \\
\hline Hypertension & $1.060 .92-1.130 .31$ & - & - \\
\hline
\end{tabular}

Abbreviations: COPD, chronic obstructive pulmonary disease; IHD, ischemic heart disease; FEV Epworth Sleepiness Scale; AHI, apnea hypopnea index, ODI, oxygen desaturation index; $\mathrm{SpO}_{2}$, pulse oxygen saturation; TST, total sleep time; TST90, percentage of total sleep time spent with oxyhemoglobin saturation below $90 \%$.

MCI from healthy controls, with $\mathrm{Se}$ and $\mathrm{Sp}$ values of $63.4 \%$ and $65.4 \%$, respectively. ${ }^{26}$ The Montreal Cognitive Assessment (MoCA), another cognitive dysfunction screening instrument, was reported to be superior to the MMSE in detecting MCI, according to two small sample-sized studies. ${ }^{2,27}$ Further studies are needed to explore more accurate and efficient assessment instruments for cognitive function among patients with COPD.
Previous studies on the relationships between lung injury and cognitive impairment among patients with COPD have yielded conflicting results. $\mathrm{Hu}$ et al found that the MMSE score is inversely correlated with the severity of airflow obstruction. ${ }^{28}$ However, Dag and Thakur examined cognitive function among stable COPD patients with the MoCA or MMSE, and they did not find any associations between COPD severity measures and the 
risk of cognitive impairment. ${ }^{16,27}$ The study conducted by Salik et al showed that cognitive function in COPD patients with mild hypoxemia was similar to that observed for healthy subjects, and no relationship existed between MMSE scores and lung function parameters in either COPD or healthy subjects. ${ }^{29}$ Similarly, in the present study, pulmonary function parameters were not associated with the risk of dementia in COPD patients with or without OSA. These discrepant consequences for cognitive function may be related to differences in disease severity and cognitive function assessment methods and to the complex interactions among COPD pathophysiological, genetic, and environmental factors. Additionally, the current result may suggest that the severity of airflow obstruction may not be closely related to the multisystem impairment of COPD, which implies the importance of comprehensive assessment of and intervention for comorbidities.

The pathophysiological mechanisms contributing to cognitive function decline in patients with COPD are complex. In addition to lung impairment, coexisting comorbidities also account for COPD-related cognitive deficits. The current data suggest that patients with both COPD and OSA had worse cognitive function than those with COPD alone. OSA has been found to adversely affect cognitive function, mainly in the attention/vigilance, executive dysfunction, verbal and visual delayed long-term memory, and visuospatial/constructional ability domains. ${ }^{30}$ In line with previous studies, we found that patients with severe OSA (AHI $\geq 30$ events/h) had worse MMSE scores than those with an AHI of $<15$ events $/ \mathrm{h}{ }^{31}$ Some studies aiming to investigate the specific role of intermittent hypoxemia and fragmented sleep in the etiology of cognitive impairment found that hypoxia seems to be more closely associated with impairments in global cognitive function, and sleep fragmentation appears to be linked to the impairment of attention/vigilance. ${ }^{8}$ In the present study, neither sleep duration nor fragmentation parameters were significantly associated with the risk of dementia in patients with COPD. This may be because the MMSE is a global measurement of cognitive function. In addition, single night in-lab PSG may not be adequately representative of usual sleep duration or quality. Cleutjens et al found that global cognitive functioning, assessed by the MMSE, was not associated with self-reported sleep disturbances in older COPD patients, which is consistent with our study. However, a trend towards worse copying function was observed in patients with sleep disturbances. ${ }^{31}$ Further studies focusing on the linkage of sleep fragmentation and the specific domains of cognitive function may provide more information. Among the hypoxia measures, the ODI was an independent predictor of the risk of dementia for patients with COPD in our study. The ODI, a marker of intermittent hypoxia, reflects the process of hypoxemia followed by reoxygenation, similar to changes in ischemic/reperfusion injury. This process may increase the formation of free radicals and inflammatory factors, which may result in damage to endothelial function and neuronal integrity. Endothelial dysfunction may further increase blood pressure and activate the coagulation process, which will increase the risk of silent stroke. ${ }^{32}$ Other measures of sleep-related hypoxemia, including the mean and lowest nocturnal $\mathrm{SpO}_{2}$ and TST90, were not found to have a significant relationship with the risk of dementia in our study. This result may suggest that intermittent hypoxia, instead of the level and duration of hypoxia, may be more closely related with a higher risk of oxidative damage and adverse outcomes. Continuous positive airway pressure (CPAP) for sleep apnea has been demonstrated to be associated with the amelioration of Alzheimer's disease (AD) neuropathological biomarkers changes in patients with subjective cognitive impairment. ${ }^{33}$ Furthermore, a recent study found that CPAP can increase gray matter volume in the hippocampal and frontal regions, which is paralleled by improvements in memory, attention, and executive functioning in patients with OSA. ${ }^{34}$ Thus, it is possible that CPAP may ameliorate cognitive decline in patients with COPD and OSA, which needs to be further studied.

It has been reported that comorbid psychological disorders, such as anxiety and depression, may increase the risk of cognitive impairment in patients with COPD. ${ }^{35}$ In the present study, we did not observe an association between the risk of dementia and anxiety or depression scores. Possible explanations may be that similar to some previously reported data, ${ }^{36}$ the HADS anxiety and depression scores in the present COPD population are relatively low, which suggests that most patients did not have significant anxiety or depression symptoms. It should also be noted that anxiety and depression symptoms were assessed by subjective questionnaires rather than by diagnosis by a psychologist in our patient population. In addition, although some studies reported that the prevalence of depression in COPD ranges from 10\%-79\%, it accounts for only a very small part (1-2\%) of the variance in cognition in these patients. ${ }^{37}$

Several limitations of this study should be noted. First, the present study investigated patients with COPD and OSA or COPD alone; however, it does not contain 
a sleep apnea only group. Although some previous studies reported lower MMSE scores in patients with OSA than in healthy controls, ${ }^{38,39}$ it is unclear whether the interaction between OSA and COPD could produce synergistic effects on cognitive decline according to the results of the present study. A normal group that has neither OSA nor COPD may also be needed, in addition to a group with OSA sans COPD, to confirm this effect. Second, the pictures of cognitive defects in COPD and OSA are very complicated, and thus far, no consensus has been achieved regarding the definition of cognitive impairment in patients with COPD. The MMSE has been proven to be an adequate test for the global evaluation of cognitive function and has been shown to be associated with important clinical outcomes of COPD. ${ }^{40}$ However, it is a relatively simple assessment for cognitive function. More detailed and specific cognitive function assessments are warranted to understand the impact of OSA on the specific domain of cognitive impairment among patients with COPD. Third, due to the open invitation for participation in the study and the lack of randomization of patients, the possibility that patients with more frequent symptoms and greater concern about having OSA and cognitive impairment may have accepted the invitation, which may have resulted in the relatively high prevalence of overlap syndrome and cognitive impairment in this sample, cannot be excluded. In addition, it should also be noted that the post hoc selection of predictor values is a limitation, as this may artificially lower the p-values in the multivariable analysis. Finally, the sample size was relatively small, and we recruited elderly patients with mainly moderate-to-severe disease severity and relatively low educational levels. As shown in previous studies and our results, age and educational level are closely related to cognitive function. ${ }^{41}$ Therefore, our findings could not be generalized to COPD patients with different severities and demographic characteristics. Future studies including more general COPD populations are needed to investigate the natural courses of cognitive decline among patients with COPD and the associated precipitating factors.

\section{Conclusions}

In summary, the current data found that cognitive impairment is common in elderly patients with COPD. Cognitive impairment is associated with an increased risk of all-cause hospitalization, disability and mortality in patients with COPD. It may be reasonable to list cognitive function assessment as a routine part of the multidimensional disease management approach because early identification of and intervention for MCI may have the potential to ameliorate the negative health care outcomes associated with cognitive impairment. Additionally, our findings showed that patients with both COPD and OSA are at elevated risk for dementia. Managing patients with these two diseases may require more individualized education and cognitive care interventions than patients with one disease. Because intermittent hypoxia is associated with cognitive impairment, future work is needed to clarify whether COPD patients with concurrent OSA can obtain cognitive function benefits from the effective treatment of the reversible disease status of sleep apnea.

\section{Acknowledgments}

The work was performed in Department of Pulmonary and Critical Care Medicine, Center of Respiratory Medicine, China-Japan Friendship Hospital, National Clinical Research Center for Respiratory Diseases, Beijing, China. This study was not involved in any off-label or investigational use, it reported an observational study, and it is not a clinical trial.

\section{Disclosure}

The authors declare that they have no known competing financial interests or personal relationships that could have appeared to influence the work reported in this paper.

\section{References}

1. Brown JP, Martinez CH. Chronic obstructive pulmonary disease comorbidities. Curr Opin Pulm Med. 2016;22(2):113-118. doi:10. 1097/MCP.0000000000000241

2. Villeneuve S, Pepin V, Rahayel S, et al. Mild cognitive impairment in moderate to severe COPD: a preliminary study. Chest. 2012;142:1516-1523. doi:10.1378/chest.11-3035

3. Dodd JW, Novotny P, Sciurba FC, Benzo RP, NETT Research Group. Executive function, survival, and hospitalization in chronic obstructive pulmonary disease. A longitudinal analysis of the National Emphysema Treatment Trial (NETT). Ann Am Thorac Soc. 2015;12:1473-1481. doi:10.1513/AnnalsATS.201506-3730C

4. Roncero C, Campuzano AI, Quintano JA, Molina J, Pérez J, Miravitlles M. Cognitive status among patients with chronic obstructive pulmonary disease. Int $J$ Chron Obstruct Pulmon Dis. 2016;11:543-551. doi:10.2147/COPD.S100850

5. Singh B, Mielke MM, Parsaik AK, et al. A prospective study of chronic obstructive pulmonary disease and the risk for mild cognitive impairment. JAMA Neurol. 2014;71:581-588. doi:10.1001/jamaneurol. 2014.94

6. Andrianopoulos V, Gloeckl R, Vogiatzis I, Kenn K. Cognitive impairment in COPD: should cognitive evaluation be part of respiratory assessment? Breathe (Sheff). 2017;13:e1-e9. doi:10.1183/20734735. 001417

7. Ouellette DR, Lavoie KL. Recognition, diagnosis, and treatment of cognitive and psychiatric disorders in patients with COPD. Int J Chron Obstruct Pulmon Dis. 2017;12:639-650. doi:10.2147/COPD.S123994 
8. Bucks RS, Olaithe M, Eastwood P. Neurocognitive function in obstructive sleep apnoea: a meta-review. Respirology. 2013;18:61-70. doi:10.1111/j.1440-1843.2012.02255.x

9. Lal C, Strange C, Bachman D. Neurocognitive impairment in obstructive sleep apnea. Chest. 2012;141:1601-1610. doi:10.1378/ chest.11-2214

10. Yaffe K, Laffan AM, Harrison SL, et al. Sleep-disordered breathing, hypoxia, and risk of mild cognitive impairment and dementia in older women. JAMA. 2011;306:613-619. doi:10.1001/jama.2011.1115

11. Bålfors EM, Franklin KA. Impairment of cerebral perfusion during obstructive sleep apneas. Am J Respir Crit Care Med. 1994;150(6 Pt 1):1587-1591. doi:10.1164/ajrccm.150.6.7952619

12. Zimmerman ME, Aloia MS. A review of neuroimaging in obstructive sleep apnea. J Clin Sleep Med. 2006;2(4):461-471. doi:10.5664/ jcsm.26665

13. Daulatzai MA. Evidence of neurodegeneration in obstructive sleep apnea: relationship between obstructive sleep apnea and cognitive dysfunction in the elderly. J Neurosci Res. 2015;93:1778-1794. doi:10.1002/jnr.23634

14. Donovan LM, Feemster LC, Udris EM, et al. Poor outcomes among patients with chronic obstructive pulmonary disease with higher risk for undiagnosed obstructive sleep apnea in the LOTT cohort. J Clin Sleep Med. 2019;15:71-77. doi:10.5664/jcsm.7574

15. Olaithe M, Bucks RS, Hillman DR, Eastwood PR. Cognitive deficits in obstructive sleep apnea: insights from a meta-review and comparison with deficits observed in COPD, insomnia, and sleep deprivation. Sleep Med Rev. 2018;38:39-49. doi:10.1016/j.smrv.2017.03.005

16. Thakur N, Blanc PD, Julian LJ, et al. COPD and cognitive impairment: the role of hypoxemia and oxygen therapy. Int $J$ Chron Obstruct Pulmon Dis. 2010;5:263-269. doi:10.2147/copd.s10684

17. Global Initiative for Chronic Obstructive Lung Disease. From the global strategy for the diagnosis, management and prevention of COPD, global initiative for chronic obstructive pulmonary disease (GOLD). 2018

18. Miller MR, Crapo R, Hankinson J, et al. General considerations for lung function testing. Eur Respir J. 2005;26(1):153-161. doi:10.1183/09031936.05.00034505.

19. Bhat S, Upadhyay H, DeBari VA, Ahmad M, Polos PG, Chokroverty S. The utility of patient-completed and partner-completed epworth sleepiness scale scores in the evaluation of obstructive sleep apnea. Sleep Breath. 2016;20:1347-1354. doi:10.1007/s11325-016-1370-8

20. Phan T, Carter O, Adams C, et al. Discriminant validity of the hospital anxiety and depression scale, beck depression inventory (II) and beck anxiety inventory to confirmed clinical diagnosis of depression and anxiety in patients with chronic obstructive pulmonary disease. Chron Respir Dis. 2016;13:220-228. doi:10.1177/ 1479972316634604

21. Mackay AJ, Donaldson GC, Patel ARC, et al. Usefulness of the chronic obstructive pulmonary disease assessment test to evaluate severity of COPD exacerbations. Am J Respir Crit Care Med. 2012;185:1218-1224. doi:10.1164/rccm.201110-1843OC

22. Crum RM, Anthony JC, Bassett SS, Folstein MF. Population-based norms for the mini-mental state examination by age and educational level. JAMA. 1993;269:2386-2391. doi:10.1001/jama.1993.03500180078038

23. Berry RB, Budhiraja R, Gottlieb DJ, et al. Rules for scoring respiratory events in sleep: update of the 2007 AASM manual for the scoring of sleep and associated events. J Clin Sleep Med. 2012;8 (05):597-619. doi:10.5664/jcsm.2172

24. Zhao YY, Blackwell T, Ensrud KE, et al. Sleep Apnea and obstructive airway disease in older men: outcomes of sleep disorders in Older Men Study. Sleep. 2016;39(7):1343-1351. doi:10.5665/ sleep. 5960
25. Cleutjens FA, Janssen DJ, Ponds RW, Dijkstra JB, Wouters EF. Cognitive-pulmonary disease. Biomed Res Int. 2014;697825. doi:10.1155/2014/697825

26. Mitchell AJ. A meta-analysis of the accuracy of the mini-mental state examination in the detection of dementia and mild cognitive impairment. $J$ Psychiatr Res. 2009;43:411-431. doi:10.1016/j. jpsychires.2008.04.014

27. Dag E, Bulcun E, Turkel Y, Ekici A, Ekici M. Factors influencing cognitive function in subjects with COPD. Respir Care. 2016;61:1044-1050. doi:10.4187/respcare.04403

28. Hu X, Wang H, Tu Y, et al. Alterations of the default mode network and cognitive impairments in patients with chronic obstructive pulmonary disease. Int J Chron Obstruct Pulmon Dis. 2018;13:519-528. doi:10.2147/COPD.S146870

29. Salik Y, Ozalevli S, Cimrin AH. Cognitive function and its effects on the quality of life status in the patients with chronic obstructive pulmonary disease (COPD). Arch Gerontol Geriatr. 2007;45 (3):273-280. doi:10.1016/j.archger.2006.12.002)

30. Wallace A, Bucks RS. Memory and obstructive sleep apnea: a meta-analysis. Sleep. 2013;36:203-220. doi:10.5665/sleep.2374

31. Cleutjens FAHM, Pedone C, Janssen DJA, et al. Sleep quality disturbances and cognitive functioning in elderly patients with COPD. ERJ Open Res. 2016;2(3):00054-2016. doi:10.1183/23120541.00054-2016

32. Yang M, Chen Y, Wu Z, et al. The impact of chronic intermittent hypoxia on the expression of intercellular cell adhesion molecule-1 and vascular endothelial growth factor in the ischemia-reperfusion rat model. Folia Neuropathol. 2018;56:159-166. doi:10.5114/fn.2018.78693

33. Liguori C, Mercuri NB, Izzi F, et al. Obstructive sleep apnea is associated with early but possibly modifiable alzheimer's disease biomarkers changes. Sleep. 2017;40(5). doi:10.1093/sleep/zsx011

34. Canessa N, Castronovo V, Cappa SF, et al. Obstructive sleep apnea: brain structural changes and neurocognitive function before and after treatment. Am J Respir Crit Care Med. 2011;183:1419-1426. doi:10.1164/rccm.201005-0693OC

35. Aras YG, Tunç A, Güngen BD, Güngen AC, Aydemir Y, Demiyürek BE. The effects of depression, anxiety and sleep disturbances on cognitive impairment in patients with chronic obstructive pulmonary disease. Cogn Neurodyn. 2017;11(6):565-571. doi:10.1007/ s11571-017-9449-x

36. Economou NT, Ilias I, Velentza L, et al. Sleepiness, fatigue, anxiety and depression in chronic obstructive pulmonary disease and obstructive sleep apnea - overlap - syndrome, before and after continuous positive airways pressure therapy. PLoS One. 2018;13(6):e0197342. doi:10.1371/journal.pone.0197342

37. Dodd JW, Getov SV, Jones PW. Cognitive function in COPD. Eur Respir J. 2010;35(4):913-922. doi:10.1183/09031936.00125109

38. Lin YN, Zhou LN, Zhang XJ, Li QY, Wang Q, Xu HJ. Combined effect of obstructive sleep apnea and chronic smoking on cognitive impairment. Sleep Breath. 2016;20(1):51-59. doi:10.1007/s11325015-1183-1

39. Xu J, Qin Z, Li W, Li X, Shen H, Wang W. Effects of somatotropic axis on cognitive dysfunction of obstructive sleep apnea. Sleep Breath. 2020;24(1):175-182. doi:10.1007/s11325-019-01854-y

40. Tulek B, Atalay NB, Yildirim G, Kanat F, Süerdem M. Cognitive function in chronic obstructive pulmonary disease: relationship to global initiative for chronic obstructive lung disease 2011 categories. Respirology. 2014;19(6):873-880. doi:10.1111/resp.12333

41. Lyketsos CG, Garrett E, Liang KY, Anthony JC. Cannabis use and cognitive decline in persons under 65 years of age. Am J Epidemiol. 1999;149:794-800. doi:10.1093/oxfordjournals.aje.a009894 
International Journal of Chronic Obstructive Pulmonary Disease

\section{Publish your work in this journal}

The International Journal of COPD is an international, peer-reviewed journal of therapeutics and pharmacology focusing on concise rapid reporting of clinical studies and reviews in COPD. Special focus is given to the pathophysiological processes underlying the disease, intervention programs, patient focused education, and self management protocols. This journal is indexed on PubMed Central, MedLine and CAS. The manuscript management system is completely online and includes a very quick and fair peer-review system, which is all easy to use. Visit http://www.dovepress.com/testimonials.php to read real quotes from published authors. 